\title{
Multiplication of the waterborne pathogen Cryptosporidium parvum in an aquatic biofilm system
}

Wan Koh ${ }^{1 *}$, Peta L Clode ${ }^{2}$, Paul Monis ${ }^{3}$ and RC Andrew Thompson ${ }^{1}$

\begin{abstract}
Background: In natural aquatic environments biofilms are known to act as environmental reservoirs for Cryptosporidium parvum oocysts. However, the fate of these oocysts within biofilms has yet to be determined.

Methods: This study aimed to identify if biofilms have the ability to support the multiplication of Cryptosporidium by measuring the change in parasite number over time using quantitative polymerase chain reaction (qPCR) and detecting the possible extracellular developmental stages using a combination of confocal microscopy and immunolabelling techniques. Pseudomonas aeruginosa biofilm flow cell systems were established and C. parvum oocysts were constantly supplied over a six day period.

Results: A significant $(P<0.001)$ increase in Cryptosporidium was detected as the biofilm matured, with the total number of C. parvum multiplying 2-3 fold during this period. With this, various Cryptosporidium developmental stages (sporozoites, trophozoites, type I and II meronts) were identified from the biofilm.

Conclusion: This is the first study demonstrating that biofilms not only serve as an environmental reservoir for oocysts, but are also capable of supporting the multiplication of Cryptosporidium over time in an aquatic environment.
\end{abstract}

Keywords: Cnyptosporidium, Biofilms, Extracellular multiplication, Water, qPCR, Fluorescence

\section{Background}

Cryptosporidium parvum is a zoonotic waterborne pathogen found worldwide [1], with Cryptosporidium oocyst levels commonly monitored in urban water distribution systems [2]. If oocysts are ingested by a suitable host, which includes humans and livestock [3], the oocyst undergoes several cycles of multiplication via both asexual (sporozoites, trophozoites) and sexual processes (merozoites and microgametes) [4]. Historically, it has always been assumed that Cryptosporidium, like other apicomplexans, can only multiply intracellularly by forming an extracytoplasmic parasitophorous vacuole in host intestinal cells and that they are not able to multiply outside of the host [5].

There are, however, an increasing number of in-vitro studies, both in cell and cell-free [6-12] cultures, demonstrating that Cryptosporidium may not be an

\footnotetext{
* Correspondence: w.koh@murdoch.edu.au

${ }^{1}$ School of Veterinary and Life Sciences, Murdoch University, South Street, Murdoch, WA 6150, Australia

Full list of author information is available at the end of the article
}

obligate intracellular parasite and can in fact multiply extracellularly. This capacity to multiply both intracellularly and extracellularly may reflect the fact that Cryptosporidium is closely related to gregarine protozoa $[9,11,13]$, which can also multiply by either means [14]. The ability of Cryptosporidium to multiply extracellularly also indicates that they are able to salvage nutrients required for multiplication from their environment, despite lacking mitochondria and associated metabolic capabilities [15]. Independent of the mechanisms, it is clear that the surrounding environment plays an important role in shaping the life cycle of Cryptosporidium.

Biofilms are highly efficient and stable ecosystems [16] that are formed mainly by bacteria [17-19], creating a favourable micro-environment that can support the survival and growth of other micro-organisms under prolonged periods of environmental stress [16]. Biofilms have been shown to serve as an environmental reservoir for Cryptosporidium oocysts in aquatic environments [20-22] and may be responsible for the occurrence of sporadic 
Cryptosporidium outbreaks [23]. Therefore, there is a current need to better understand Cryptosporidium behaviour in biofilm environments, especially in relation to water distribution systems $[18,22]$ and to investigate whether Cryptosporidium oocysts captured within biofilms can utilise this nutrient rich micro-environment to survive and multiply.

The aim of this study was to investigate whether biofilms can support the multiplication of Cryptosporidium in aquatic environments. Model flow cell biofilm systems were developed and confocal laser scanning microscopy coupled with image analysis was used to quantitatively compare biofilm thickness between pure biofilm cultures and biofilms exposed to Cryptosporidium oocysts. Quantitative PCR and a combination of confocal microscopy and immunolabelling were used to monitor Cryptosporidium within the biofilm system over a six day period.

\section{Methods}

\section{Bacterial strains and media}

Wild type Pseudomonas aeruginosa bacteria (PA01) were used to establish the biofilms for this study. Before incubating into the biofilm flow cell system, Pseudomonas cultures were maintained on Pseudomonas agar (BeckonDickson) at $37^{\circ} \mathrm{C}$. A $10 \%$ solution of tryptic soy broth (Beckon-Dickson) was used as the flow-through media in all biofilm experiments.

\section{Parasite isolation and purification}

C. parvum cattle genotype (Swiss cattle C26) oocysts were obtained from the Institute of Parasitology, University of Zurich and were subsequently passaged through and purified from ARC/Swiss mice as described by Meloni and Thompson [24]. Purified oocysts were stored in $1 \mathrm{x}$ phosphate buffered saline (PBS) with antibiotics (10,000 U penicillin G and $0.01 \mathrm{~g}$ streptomycin; Sigma) at $4^{\circ} \mathrm{C}$ before use. Oocysts used in biofilm experiments were less than 4 weeks old and were decontaminated with $2 \%$ household bleach at room temperature. Identical batches of oocysts were used for parallel control experiments.

\section{Flow cell biofilm systems}

Flow cell biofilm systems were set up as described by Werner et al. [25], except that here, the system was modified to be a fully closed system with no air intake, to prevent air contamination (Figure 1). Instead, two capillary flow cells $(20 \mathrm{~cm}$ in length) were run in parallel, and a silicon tube was attached between the influent and effluent to ensure a smooth air flow. Hence, no bubbles were observed in the liquid flow. Five-litre glass bottles were used for both influent and effluent media. All experiments were performed at room temperature under sterile and dark environmental conditions.

$P$. aeruginosa inoculum with a turbidity equivalent to that of one McFardland standard was prepared from Pseudomonas agar and $1 \mathrm{ml}$ of inoculum was transfered to the flow cells as described by Werner et al. [25]. To allow bacterial attachment to the surface of the flow tube, no flow was initiated for the first $24 \mathrm{~h}$. Decontaminated oocysts were injected into the influent medium and the flow initiated and continued $\left(60 \mathrm{ml} \mathrm{h}^{-1}\right)$ for one, three or six days. Biofilms exposed to Cryptosporidium oocysts are hereafter referred to as Cryptosporidium-exposed biofilm samples.

The volume of influent medium and the number of introduced oocysts were adjusted according to the duration of the experiment (number of days). The experiment was designed such that 51 of $10 \%$ tryptic

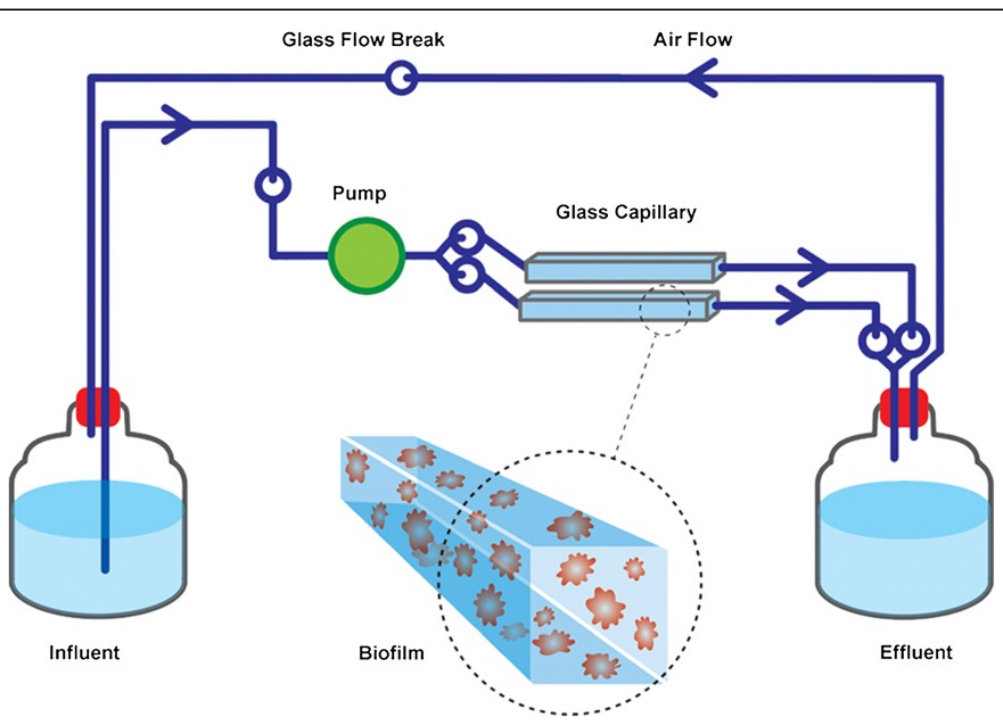

Figure 1 Schematic diagram of the capillary flow cell biofilm system used in this study. System and picture modified from Werner et al. [25]. 
soy broth was sufficient for a three-day experiment. The number of oocysts introduced in the influent medium was calculated so that the biofilms received $1 \times 10^{6}$ oocysts every $24 \mathrm{~h}$. In order to avoid air contamination, the oocysts were introduced at the beginning of the experiment (for all experiments), and during an influent medium change after three days (for the six day experiment). Two controls were set up simultaneously:

1) No biofilm was developed. Decontaminated oocysts were added into the flow system at similar rates, but without any biofilm established within the flow system. This sample is presented as the biofilm-free control.

2) No oocysts were added. Biofilms were grown in the flow system, without the introduction of Cryptosporidium oocysts. This sample is presented as the biofilm-only control.

All experiments utilised two flow tubes simultaneously for each treatment and were repeated three times.

\section{Biofilm thickness}

Film Tracer calcein red orange (Invitrogen, Australia) was used to stain the biofilm for confocal laser scanning microscopy imaging and analysis. The biofilms were stained according to the protocol provided with the stain. Briefly, calcein red orange stain was introduced into the living biofilm within the flow tube using a peristaltic pump at a rate of $60 \mathrm{ml} \mathrm{h}^{-1}$ and incubated in the dark for $60 \mathrm{~min}$ at room temperature. Calcein red orange stain was similarly flushed from the system and the biofilms incubated with sterile water. Confocal images of biofilms were acquired from the flow cells directly using $576 \mathrm{~nm}$ and $590 \mathrm{~nm}$ laser excitation/emission wavelengths. Average biofilm thickness was determined from three regions - the left, right and middle area - of the flow cell. For both biofilm-only and Cryptosporidium-exposed biofilms, serial sections in the $x y$ plane were obtained at $0.44 \mu \mathrm{m}$ (one day old biofilm) and 2-10 $\mu \mathrm{m}$ intervals (three and six day old biofilms) along the $z$-axis, and the $z$-stack image was then analysed with COMSTAT II [26].

\section{DNA extraction}

Following thickness measurements, biofilms were dispersed by incubation in $500 \mathrm{nmol} \mathrm{l}^{-1}$ sodium nitriporusside (Sigma Aldrich, NSW, Australia) overnight, after which the flow cells were gently washed with $1 \mathrm{x}$ PBS several times to completely detach the biofilms from the flow cell surface [27]. The cell suspensions were further washed several times with $1 \mathrm{x}$ PBS to remove any residual nitric oxide. Biofilms from the flow cell were resuspended in $1 \mathrm{x}$ PBS to a final volume of $400 \mu \mathrm{l}$. To quantify the total number of parasites present at the end of each experimental period, the dispersed biofilms from the effluent were also collected and resuspended to a final volume of $2 \mathrm{ml}$.

Aliquots of flow cell biofilms $(100 \mu \mathrm{l})$ and effluent biofilms $(500 \mu \mathrm{l})$ were used for DNA extraction. The freeze-thaw DNA extraction method was used for all samples with the process being repeated 12 times to release DNA from Cryptosporidium. The resulting DNA suspension was further purified as described by the Promega Genomic DNA extraction kit protocol, except that the cell lysis step was modified from 15 min to 30 min incubation to allow complete lysing of biofilm aggregates. The purified DNA was eluted in a final volume of $50 \mu \mathrm{l}$ in the DNA suspension buffer provided with the kit.

\section{Quantitative polymerase chain reaction (qPCR)}

The DNA-based technique of quantitative polymerase chain reaction (qPCR) was utilised to quantify the numbers of Cryptosporidium within one, three and six day old biofilms. All qPCR reactions were performed on a Qiagen Rotor Gene 2 system. Standard curves were constructed using five genomic DNA triplicates extracted from a known number of oocysts and serially diluted at 1:10 dilution ratio, calibrated to correspond from $10^{0}$ to $10^{5}$ oocysts. $C$. parvum specific primers used in this study were designed to target the glyceraldehydes-3-phosphate dehydrogenase (GAPDH) gene. The qPCR reactions contained $12.5 \mu \mathrm{l}$ of Promega GoTaq SYBR Green master mix, $2.5 \mathrm{mmol} \mathrm{l}^{-1}$ of bovine albumin serum, $10 \mu \mathrm{mol} \mathrm{l}^{-1}$ primers specific for $C$. parvum (forward primer 5'ATCAAGCCGTTAAGGAA GCA3', reverse primer 5'AAATCGGTCGAGACGACA TC3') and $5 \mu$ of DNA to a final volume of $25 \mu \mathrm{l}$. Thermal cycling was performed at $95^{\circ} \mathrm{C}$ for $2 \mathrm{~min}$ to activate Go-Taq polymerase then the amplification and annealing conditions involved 40 cycles of $15 \mathrm{~s}$ at $94^{\circ} \mathrm{C}$ and 1 minute at $60^{\circ} \mathrm{C}$. Data was collected during the annealing step of each cycle. After amplification, a melting curve analysis was performed to determine the specificity of the PCR product. The PCR products were incubated for $15 \mathrm{sec}$ at $55^{\circ} \mathrm{C}$ and the temperature was increased to $95^{\circ} \mathrm{C}$ with a ramp rate of $0.1^{\circ} \mathrm{C} \mathrm{sec}{ }^{-1}$.

As the concentration of DNA in the biofilm samples was too high, DNA dilution was performed to increase the qPCR efficacy of detecting Cryptosporidium in the biofilm system. Generally, the dilution factors for detecting Cryptosporidium were 1:100 in one and three day old Cryptosporidium-exposed biofilms and 1:1000 for six day old Cryptosporidium-exposed biofilms. Biofilm-free and six day old biofilm-only samples (negative controls) were diluted according to the respective dilution factor of the biofilm waste samples. The total number of Cryptosporidium present in each experiment was obtained by adding the number of parasites within the biofilm and the number of parasites within the effluent from each experiment. Biofilm-free controls were similarly analysed to provide a 
comparable baseline of the number of Cryptosporidium parasites within the system, independent of the biofilm.

\section{Statistical analyses}

All experimental data are presented as mean \pm the standard error of the mean. Analyses were performed using GraphPad Prism, version 5 (GraphPad Software, San Diego, CA). One-way ANOVA analysis of variance was performed to determine whether there were significant differences in (i) thickness between one, three and six day old biofilm only controls (ii) thickness between one, three and six day old Cryptosporidium-exposed biofilms, (iii) amount of Cryptosporidium parasites detected from one, three and six day old Cryptosporidium-exposed biofilms (flow cell). Two-way ANOVA analysis of variance was used to determine whether there were significant differences in (i) thickness between Cryptosporidium-exposed biofilms and biofilm-only controls, (ii) the total number of Cryptosporidium detected at the completion of experimental and biofilm-free experiments.

\section{Immunolabelling}

As a significant increase in Cryptosporidium DNA was detected in six day old Cryptosporidium-exposed biofilms, immunolabelling with Cryptosporidium-specific Sporo-a-glo antibody was performed on this sample to examine the possible Cryptosporidium developmental stages present within the biofilm. This antibody has been shown to label various Cryptosporidium developmental stages such as sporozoites, trophozoites, type I/II meronts and type I/II merozoites $[7,28,29]$. An aliquot of six day old dispersed biofilm suspension $(20 \mu \mathrm{l})$ was fixed with $2.5 \%$ paraformaldehyde in PBS for $20 \mathrm{~min}$ at $4^{\circ} \mathrm{C}$. The cells were pelleted and incubated with $200 \mu \mathrm{l}$ of blocking buffer (6\% BSA $+10 \%$ rat serum in $1 \times \mathrm{PBS})$ for $1 \mathrm{~h}$ at room temperature, then washed with $\mathrm{PBS}$ ( $\mathrm{pH}$ 7.4, 2 times, $5 \mathrm{~min}$ ) and incubated with polyclonal primary antibody, Sporo-a-glo (Waterborne Inc) (1:100 in blocking buffers) for $2 \mathrm{~h}$ at $37^{\circ} \mathrm{C}$. The samples were then washed again with PBS (twice for $6 \mathrm{~min}$ ) and incubated with $40 \mathrm{nmol} \mathrm{l}^{-1}$ quantum dot 655 anti-rat secondary antibody (catalogue no Q-11621MP, Invitrogen) for $2 \mathrm{~h}$ at room temperature. The cells were then rinsed 3 times with $1 \times$ PBS and resuspended to a final volume of $100 \mu \mathrm{l}$. For comparison, unexcysted oocysts and six day old biofilm-only samples were also similarly stained.

Experimental controls included six day old Cryptosporidium-exposed biofilms and excysted Cryptosporidium oocysts labelled with (i) primary antibody only or (ii) secondary antibody only.

\section{Confocal microscopy}

For examination of Cryptosporidium biofilms samples by confocal microscopy, an aliquot of the immunolabeled suspension was immobilised and attached to coverslips using $0.01 \%$ poly-L-lysine (Sigma, USA). Prior to coverslip coating, coverslips were rinsed in 100\% ethanol to remove surface dirt and any contamination and air dried in a laminar hood for $20 \mathrm{~min}$. Coverslips were coated with polyL-lysine for $20 \mathrm{~min}$ at room temperature and were then washed with sterile water to remove excess poly-L-lysine. After being air dried in the laminar flow, the experimental sample was then transferred onto the coverslip for attachment and incubated in a humidified box for $20 \mathrm{~min}$ to prevent dehydration. The sample was then analysed directly by confocal microscopy (Leica SP2 inverted) using the excitation and emission wavelength of FITC at $488 / 525 \mathrm{~nm}$. Oil immersion was used for magnifications above 40x.

\section{Ethical approval}

The animal work in this study was reviewed and approved by the Animal Ethics Committee of Murdoch University, Australia (Permit number: R2310/10).

\section{Results}

Comparison of biofilm thickness: biofilm-only vs. Cryptosporidium-exposed biofilm

Biofilm thickness in biofilm-only and Cryptosporidiumexposed biofilm experiments was determined using COMSTAT II [26]. For biofilm-only experiments, one day old biofilms were found to be $0.7 \pm 0.4 \mu \mathrm{m}$ thick (Figure 2), indicating that the biofilm was still developing and could

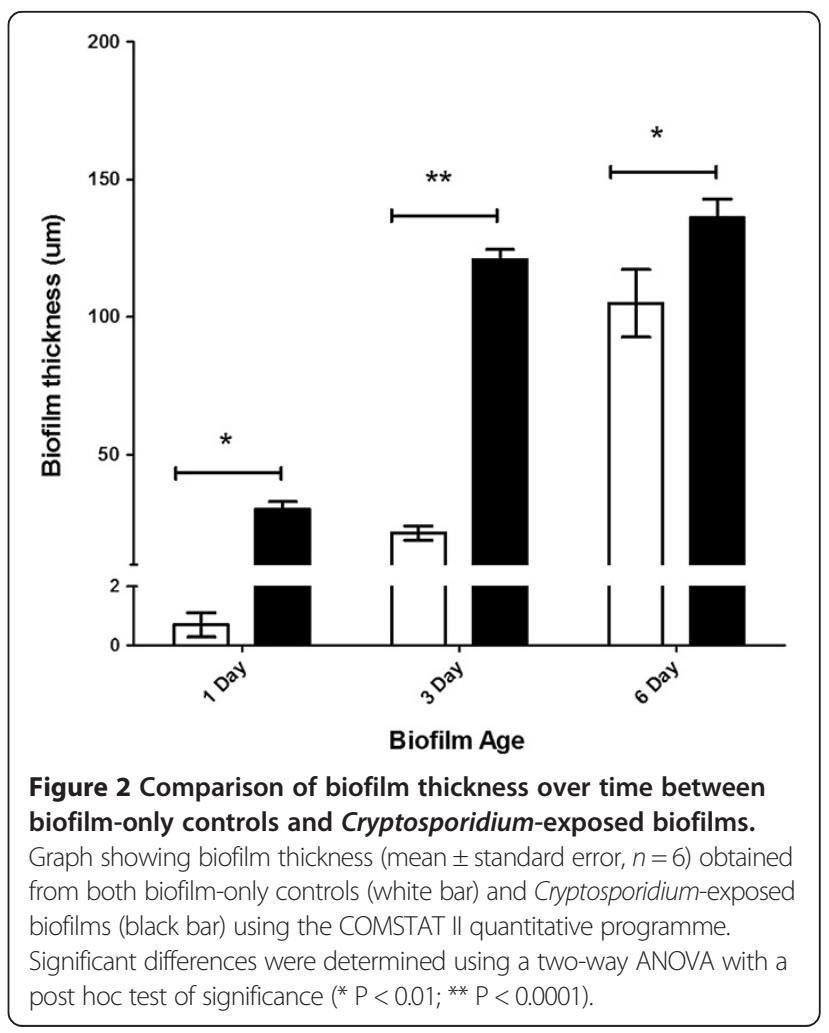


therefore be described as an immature biofilm. After three days the thickness of the biofilm had increased to $21 \pm 3 \mu \mathrm{m}$, but this was not significantly different $(\mathrm{P}>0.05)$ from the one day old biofilms (Figure 2). However, a significant $(\mathrm{P}<0.05)$ increase in biofilm thickness was observed in six day old biofilms $(105 \pm 12 \mu \mathrm{m})$ (Figure 2). This biofilm thickness fulfills the definition of a mature biofilm as defined by Davies et al. [30], and hence was considered a mature biofilm.

When Cryptosporidium oocysts were introduced into the biofilm system, one day old Cryptosporidiumexposed biofilms formed immature biofilms that were $30 \pm 3 \mu \mathrm{m}$ thick (Figure 2). Unlike biofilm-only experiments, a significant $(\mathrm{P}<0.05)$ increase in biofilm thickness was observed in Cryptosporidium-exposed biofilms after only three days $(121 \pm 4 \mu \mathrm{m})$ (Figure 2$)$. The thickness of these three day old Cryptosporidium-exposed biofilms $(\sim 100 \mu \mathrm{m})$ could be classified as the penultimate stage of biofilm development $[30,31]$ and was considered to be a mature biofilm. No significant $(P>0.05)$ increase in the thickness of this mature biofilm was observed from day three to day six in Cryptosporidium-exposed biofilms, with six day old biofilms found to be $136 \pm 7 \mu \mathrm{m}$ thick (Figure 2).

All Cryptosporidium-exposed biofilms were thicker than biofilm-only controls, but the level of significance varied. Although both biofilm-only controls and Cryptosporidium-exposed biofilms formed immature biofilms on day one and mature biofilms by day six, Cryptosporidium-exposed biofilms were always significantly $(\mathrm{P}<0.01)$ thicker than the biofilm-only controls. In addition, by day three, Cryptosporidium-exposed biofilms had formed mature biofilms and thus had matured significantly $(\mathrm{P}<0.0001)$ faster than the comparable three day old immature biofilms in the biofilm-only control.

\section{Association of Cryptosporidium with biofilms}

Overall, qPCR revealed that the number of Cryptosporidium parasites retained within the biofilm increased significantly $(\mathrm{P}<0.05)$ (Figure 3$)$ and continually over time, as the biofilm increased in thickness and matured. When one and three day old Cryptosporidium-exposed biofilms were compared, a significant $(\mathrm{P}<0.05)$ increase in both parasite number (Figure 3) and biofilm thickness (Figure 2) was observed over time. However, even when the Cryptosporidium-exposed biofilm had reached maturity and no longer underwent any increase in thickness (day three) (Figure 2), a significant $(\mathrm{P}<0.05)$ increase in parasite numbers continued to be observed until day 6 (Figure 3).

Similarly, for the total number of parasites in the flow system (i.e. both those retained within the biofilm + those recovered from the effluent), an increase in the total number of Cryptosporidium was observed in both one

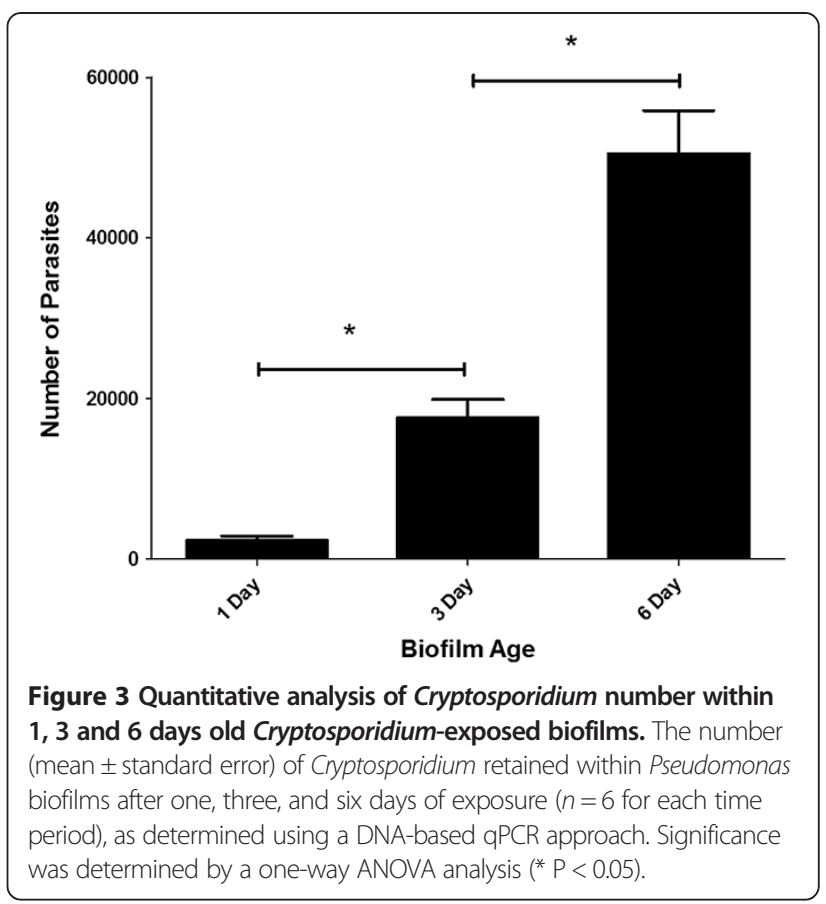

and three day old Cryptosporidium-exposed biofilms, but these were not significantly $(\mathrm{P}>0.05)$ different to biofilmfree controls (Figure 4). However, after six days, Cryptosporidium-exposed biofilms contained 2-3 fold more Cryptosporidium than that of the biofilm-free controls,

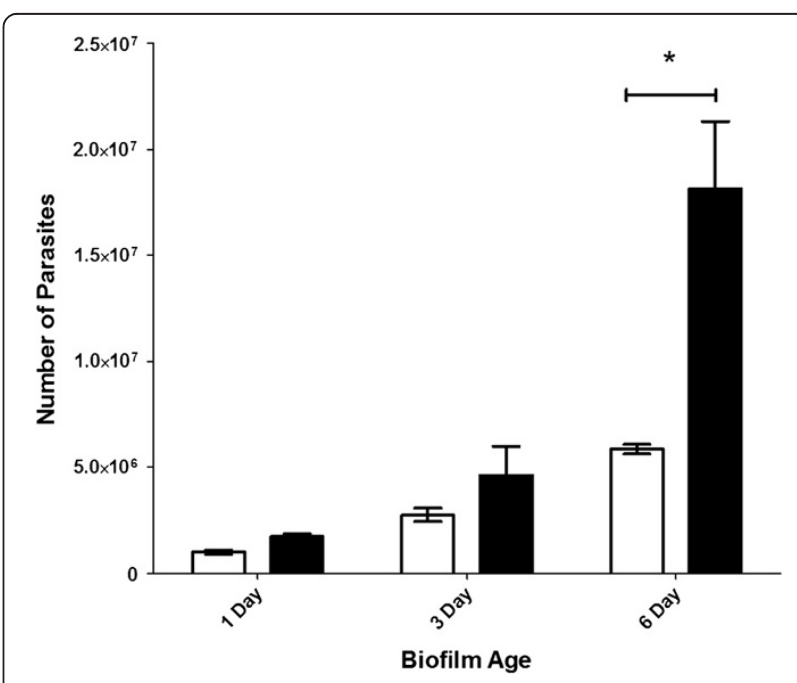

Figure 4 Comparison of the total number of Cryptosporidium present in Cryptosporidium-exposed biofilms and biofilm-free control systems. Graph showing the total number

(mean \pm standard error) of Cryptosporidium (retained in biofilm + effluent) determined at the end of each experiment (one, three and six day) for Cryptosporidium-exposed biofilms (black bar) $(n=3)$ and Cryptosporidium only (biofilm-free) control systems (white bar) $(n=3)$, as determined using a DNA-based qPCR approach. Significance was determined by a two-way ANOVA analysis with a post hoc test of significance ( $\left.{ }^{*} P<0.001\right)$. 
which reflected a highly significant $(\mathrm{P}<0.001)$ increase in parasite numbers when a biofilm was present (Figure 4). The significant increase in Cryptosporidium numbers within the biofilm flow system over the six day period implies that Cryptosporidium was not simply captured and accumulated but underwent multiplication within the biofilm system.

\section{Confocal microscopy}

The six day old Cryptosporidium-exposed biofilm sample (flow cell) was further analysed using confocal microscopy to examine the possible developmental stages of Cryptosporidium present within the biofilm.
Clusters of Cryptosporidium and bacteria cells were frequently observed after biofilm dispersion, thus we differentiated Cryptosporidium from the bacterial biofilm using specific fluorescence immunolabelling of Cryptosporidium developmental stages. Oocyst excystation was observed to have occurred with the released of slender shaped sporozoites (Figure 5A). Comma-shaped sporozoites with a rounded posterior end and a pointed tapered anterior end were also visualised, indicating that trophozoite transformation had begun (Figure 5B). Circular shaped $(2 \times 2 \mu \mathrm{m})$ trophozoites were identified indicating completion of the trophozoite transformation process (Figure 5C). Trophozoites represent a transitional stage
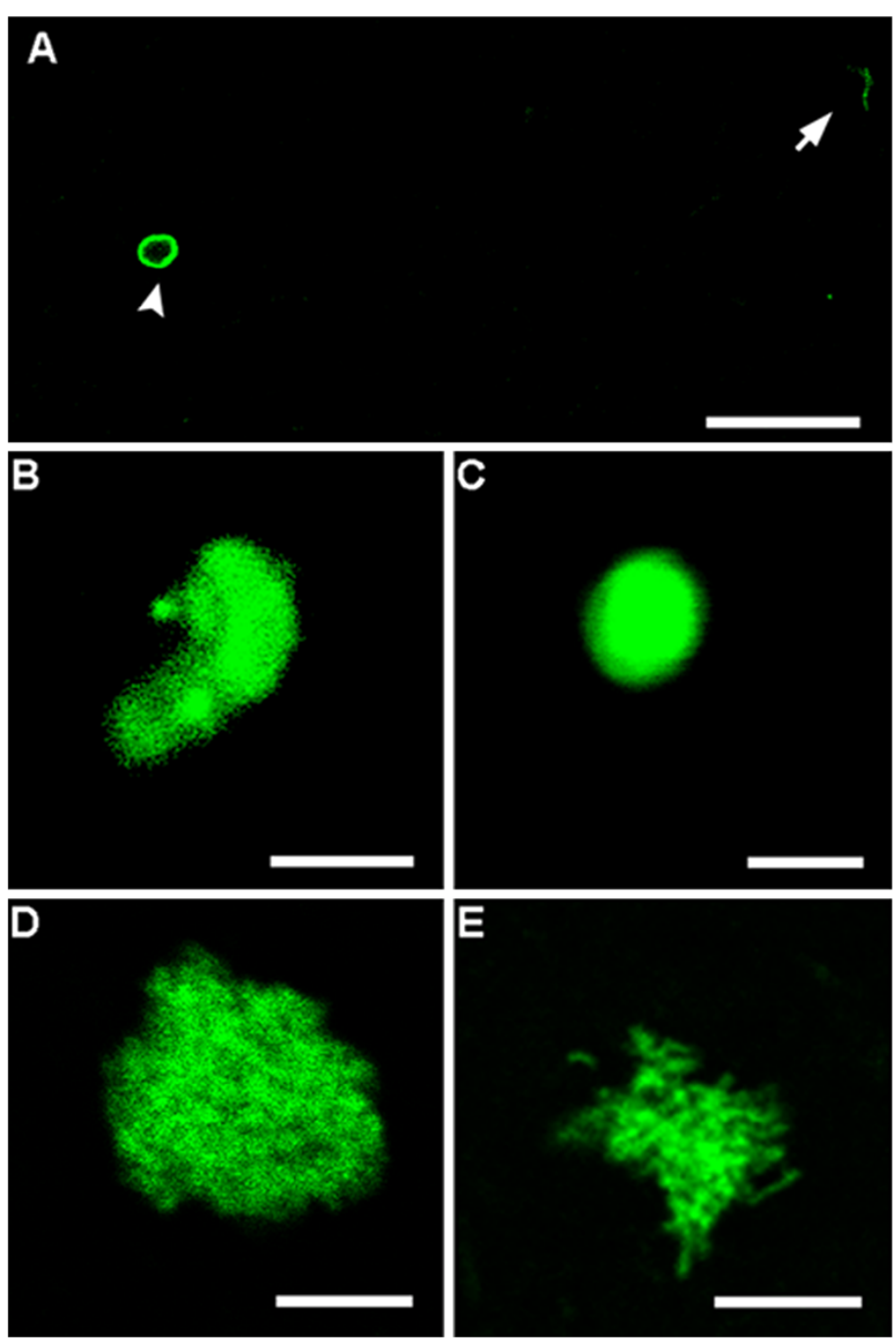

Figure 5 Confocal observation of Cryptosporidium extracellular developmental stages identified within six day old Cryptosporidium-exposed biofilms. A) excysted oocyst (indicated by arrowhead) with newly released slender sporozoite (indicated by arrow). B) comma shaped sporozoite; C) circular-shaped trophozoite; D and E) types I and II meront respectively. Scale bars: $\mathbf{A}: 20 \mu \mathrm{m} ; \mathbf{B} \& \mathbf{C}: 2 \mu \mathrm{m} ; \mathbf{D} \& \mathbf{E}: 5 \mu \mathrm{m}$. 
from sporozoites and merozoites to meronts [3]. In addition to trophozoites, two different types of meronts similar to those description by Hijjawi et al. [8] were also observed (Figure 5D-E). The grape-like aggregated merozoites (small with circular to oval shapes, approximately $1 \times 1 \mu \mathrm{m}$ ) conform to type I meront (Figure 5D) while the aggregation of many spindle-shaped merozoites with pointed ends conform to type II meront $(0.5 \times 1 \mu \mathrm{m})$ (Figure $5 \mathrm{E})$. The presence of type I/II meronts indicated that Cryptosporidium gametogony process had been initiated.

No fluorescent signal was observed in the control samples (six day old Cryptosporidium-exposed biofilms and excysted oocysts) where either the primary or the secondary antibody had been omitted (Figures 6 and 7). Fully labelled six day old biofilm-only control samples also displayed no fluorescence (Figure 8A-B). In addition, Sporo-a-glo, which is a Cryptosporidium-specific developmental stage antibody, binds only very weakly to unexcysted oocysts (Figure 8C-D). From this, we conclude that the abundant fluorescent expression observed within the biofilm samples derives from actively growing Cryptosporidium, rather than simply from accumulated unexcysted or degenerate oocysts. These observations are consistent with the qPCR data and show that oocysts were not simply retained within the biofilms, and had undergone the multiplication process extracellularly, as evidenced by the observation of several developmental and transitional stages.

\section{Discussion}

This is the first study to demonstrate a significant increase in Cryptosporidium numbers over time within a biofilm system, highlighting that biofilms can readily provide a suitable environment for not only the retention, but also the multiplication of Cryptosporidium parasites, in aquatic environments. Previous studies [32,33] have shown that the number of oocysts retained within biofilms remained constant while oocysts were continually supplied to the biofilm system. However, the apparent discrepancy between these results and those presented here can be explained by the difference in the type of biofilm and methods used to detect Cryptosporidium. In studies by Wolyniak et al. [32,33], natural biofilms were used with filter sterilised creek water used as the medium. Therefore, when compared to our artificial Pseudomonas biofilms, their biofilms would have a different community structure and nutrient levels. In addition, through the use of the qPCR technique, our analyses quantified not only the oocysts within the system
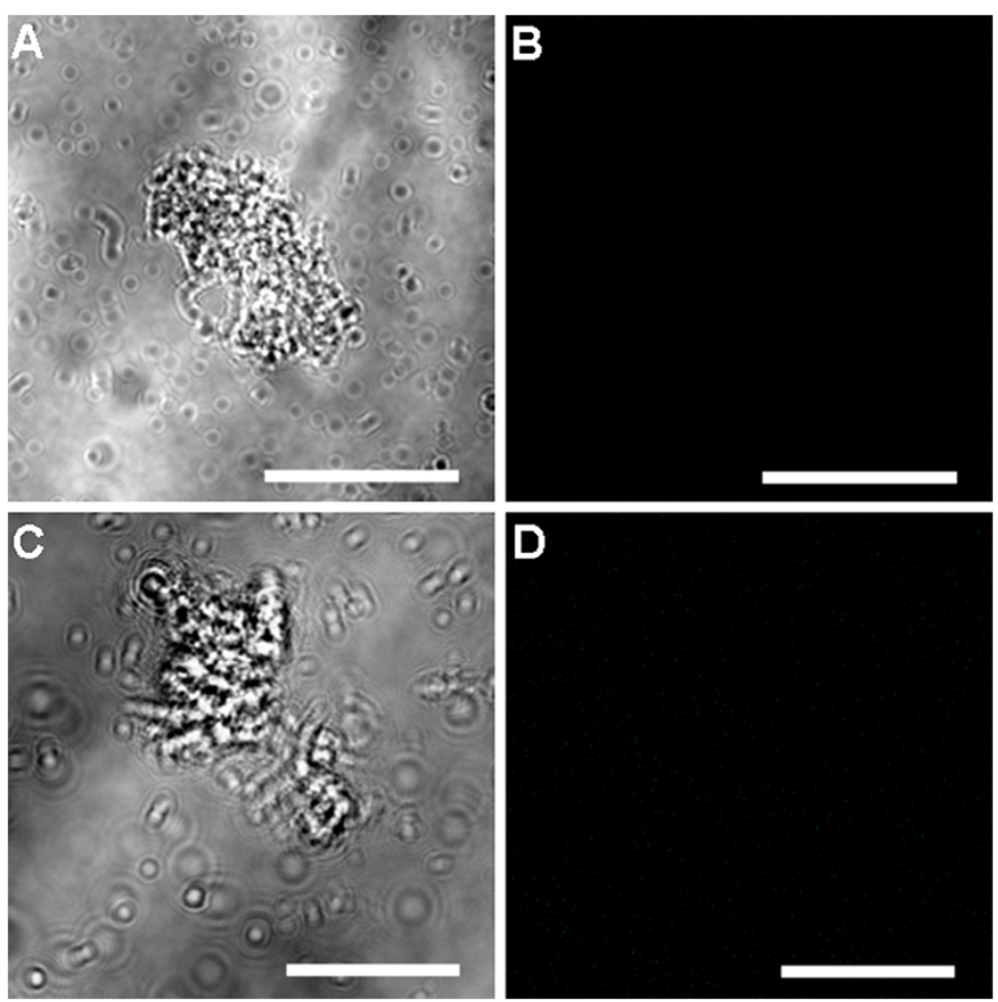

Figure 6 Confocal observation of six day old Cryptosporidium-exposed biofilm control samples. A) Six day old Cryptosporidium biofilms labelled with only primary antibody seen under transmitted light; B) corresponding confocal image, showing no immunolabeling. C) Six day old Cryptosporidium biofilms labelled with only secondary antibody seen under transmitted light; D) corresponding confocal image, showing no immunolabeling. Scale bars: A\&B: $50 \mu \mathrm{m} ; \mathbf{C} \& \mathbf{D}: 20 \mu \mathrm{m}$. 

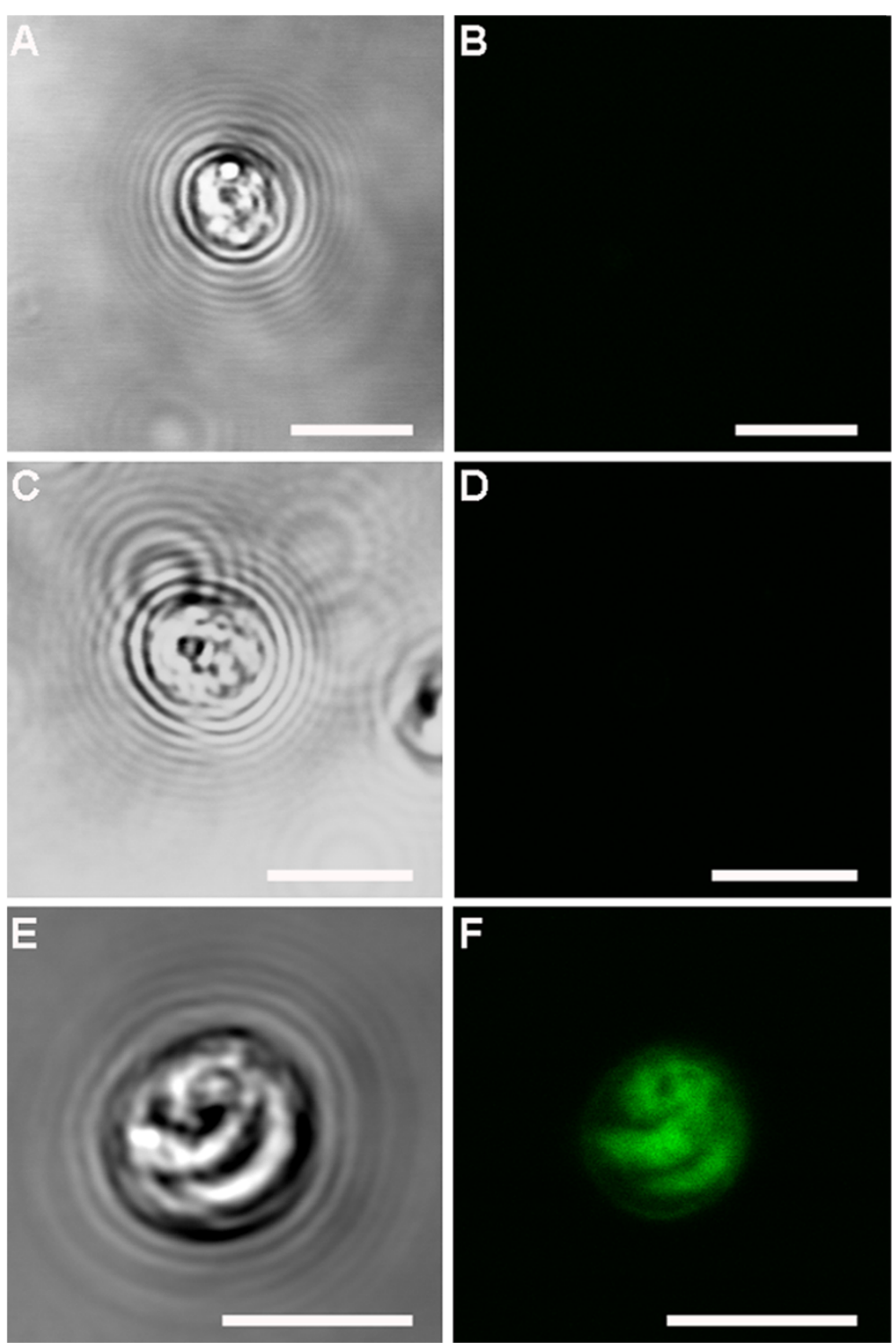

Figure 7 Confocal observation of excysted oocysts control samples. A) excysted oocyst labelled with only primary antibody seen under transmitted light; B) corresponding confocal image, showing no immunolabeling. C) excysted oocyst labelled with only secondary antibody seen under transmitted light; D) corresponding confocal image, showing no immunolabeling. E) excysted oocyst labelled with both primary and secondary antibodies seen under transmitted light; F) corresponding confocal image, showing oocyst intensely immunolabelled. Scale bars: $5 \mu$ m.

but also other Cryptosporidium life stages that were produced through multiplication.

The significant increase in Cryptosporidium DNA in six day old Cryptosporidium exposed biofilm was further supported by confocal microscopy observation. Cell free culture studies by Hijjawi et al. [7,8], Karanis et al. [10] and Zhang et al. [12] support our observation that Cryptosporidium can multiply extracellularly, and that encapsulation within a host cell is not essential for multiplication to occur. Due to the difficulty of identifying Cryptosporidium from a large background of bacteria from biofilms, not all Cryptosporidium stages were identified.
Nevertheless, several key developmental stages representing both asexual and sexual reproduction were observed, including sporozoites, trophozoites, and types I and II meronts. The observation of morphological changes of Cryptosporidium sporozoites agrees with previous in-vivo and in-vitro culture observations [34-36], including sporozoites becoming oval shaped during the trophozoite transformation process. Although previous studies by Petry et al. [37] and Matsubayashi et al. [38] suggested these changes were due to aged sporozoites that could not multiply in the nutrient-limited cell free culture environment, our observation of subsequent 

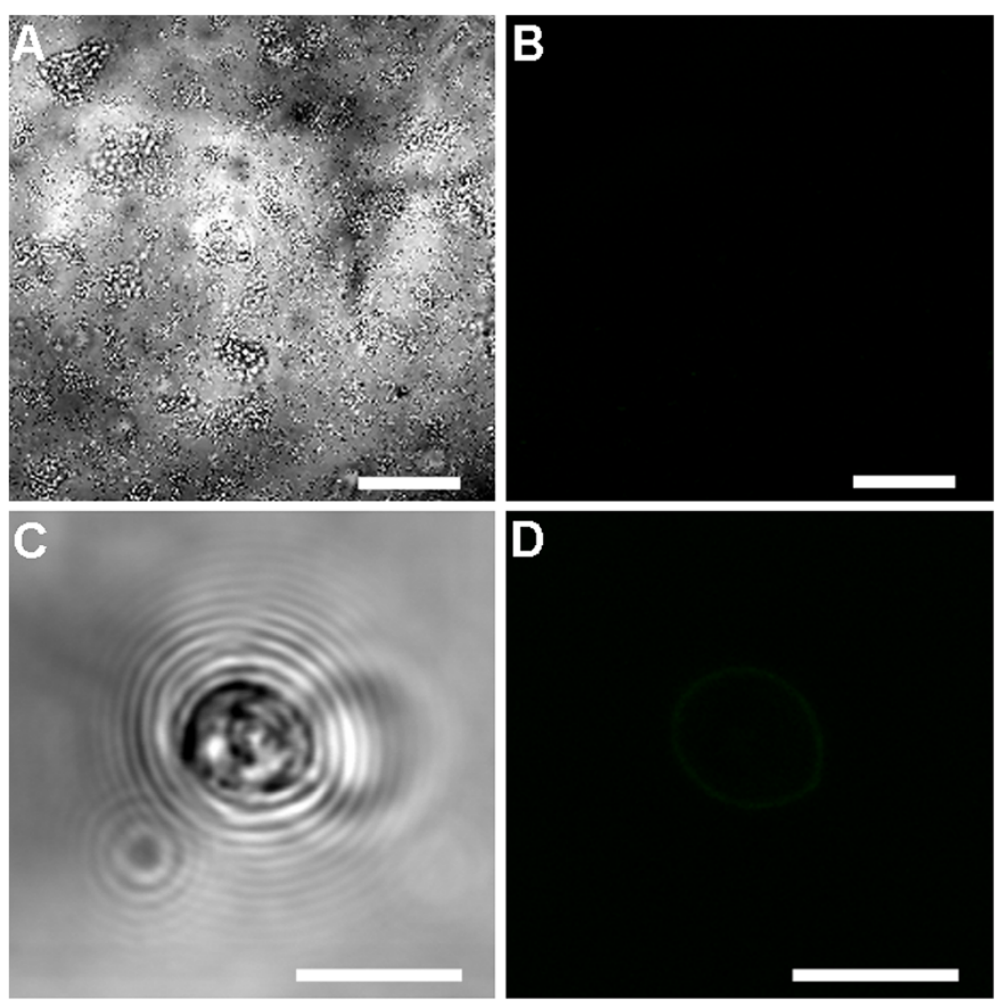

Figure 8 Confocal observation of unexcysted oocyst and six day old biofilm-only control samples. A) biofilm-only labelled with both primary and secondary antibodies seen under transmitted light; B) corresponding confocal image, showing no immunolabeling. C) Unexcysted oocyst labelled with both primary and secondary antibodies seen under transmitted light; D) corresponding confocal image, showing no immunolabeling Scale bars: $\mathbf{A} \& \mathbf{B}: 50 \mu \mathrm{m}$; C\&D: $5 \mu \mathrm{m}$.

Cryptosporidium development stages of the life cycle, such as types I and II meronts (based upon the developmental stage descriptions by Hijawi et al. [8]) within six day old biofilm provides evidence that these sporozoites were not simply aged sporozoites. The misinterpretations by these authors $[37,38]$ have also been clearly defended and clarified by Karanis and Aldeyarbi [39]. Furthermore, the environments that liberated sporozoites are exposed to in aquatic biofilms are nutrient rich micro-environments that could allow Cryptosporidium to salvage their metabolite needs to fuel their high rate of growth and multiplication. This ability to multiply either intracellularly or extracellularly [6,8-12] suggests that Cryptosporidium i) is capable of extracting the nutrients required for growth and multiplication from the surrounding environment, ii) is not an obligate intracellular parasite, and iii) may be physiologically as well as genetically similar to the closely-related gregarines [40,41]. Further high resolution imaging $[6,29,42,43]$ and flow cytometry-based studies [44-46] are now needed to fully characterise any additional life stages that may be produced within biofilms.

Interestingly, the presence of Cryptosporidium was also shown to significantly affect biofilm development and maturation. Cryptosporidium-exposed biofilms were found to form mature biofilms significantly faster than biofilms forming without exposure to Cryptosporidium. Consistent with this, Singleton et al. [47] also showed that biofilms that contain both prokaryotic and eukaryotic cells often formed extensive dense and thick mature biofilms. As the biofilm sloughs off after growing to a certain density, we are unable to conclusively determine if Cryptosporidium detected in the effluent was a result of cells sloughing off with the biofilm, or free floating cells. It is likely to be both. Although it is not possible to determine what proportion of the increase in the mature biofilm thickness was due to increases in the number of bacteria or of Cryptosporidium, our qPCR analyses demonstrated that even very immature biofilms were capable of capturing and accumulating Cryptosporidium oocysts. These may be incidentally incorporated into cell clusters during the biofilm aggregation process. Additionally, during transformation from an immature to a mature biofilm, matrix and water channels that form on the biofilm surface may have enhanced the adhesion of oocysts and also encased both parasites and bacteria, trapping those that were already retained within the biofilm [32,48]. However, while the thickness and maturation rate of the biofilm was affected by Cryptosporidium, the two factors were 
not strongly correlated, thus biofilm thickness cannot be reliably used as an indicator of the number of Cryptosporidium residing within the biofilm, a finding also concluded by other studies $[32,33,49,50]$.

\section{Conclusion}

In conclusion, this study shows that biofilms not only serve as an environmental reservoir for oocysts, but are also capable of supporting Cryptosporidium multiplication in an aquatic environment. The presence of biofilm on the pipes of water systems may pose a public health threat as Cryptosporidium residing within biofilms may increase in quantity over time, before being released into the water supply. With this, authorities should take into consideration the ability of Cryptosporidium to multiply within biofilms in aquatic environments when designing preventive measures to control Cryptosporidium contamination in water distribution systems.

\section{Competing interests}

The authors declare that they have no competing interests.

\section{Authors' contributions}

WK, PLC, PM and RCAT designed the study; WK, PLC, PM and RCAT implemented the study; WK managed the data; WK, PLC and RCAT analysed and interpreted the data; WK wrote the paper. WK, PLC, and RCAT supervised the different phases of the study. All authors read, revised and approved the final manuscript.

\section{Acknowledgements}

The authors thank the Australian Research Council for financial support and acknowledge the facilities, and the scientific and technical assistance of the Australian Microscopy \& Microanalysis Research Facility at the Centre for Microscopy, Characterisation \& Analysis, The University of Western Australia, a facility funded by the University, State and Commonwealth Governments. We also thank Mr. John Murphy for the confocal microscopy technical assistance.

\section{Author details}

'School of Veterinary and Life Sciences, Murdoch University, South Street, Murdoch, WA 6150, Australia. ${ }^{2}$ Centre for Microscopy, Characterisation and Analysis, The University of Western Australia, 35 Stirling Hwy, Crawley, WA 6009, Australia. ${ }^{3}$ South Australian Water Corporation, 250 Victoria Square, Adelaide, SA 5000, Australia.

Received: 6 August 2013 Accepted: 15 September 2013

Published: 19 September 2013

\section{References}

1. Putignani $L$, Menichella D: Global distribution, public health and clinical impact of the protozoan pathogen Cryptosporidium. Interdiscip Perspect Infect Dis 2010. doi: 10.1155/2010/753512.

2. Rose JB: Environmental ecology of Cryptosporidium and public health implications. Annu Rev Public Health 1997, 18:135-161.

3. Thompson RCA, Olson ME, Zhu G, Enomoto S, Abrahamsen MS, Hijjawi NS Cryptosporidium and cryptosporidiosis. Adv Parasitol 2005, 59:77-158

4. Borowski $\mathrm{H}$, Clode PL, Thompson RCA: Active invasion and/or encapsulation? A reappraisal of host-cell parasitism by Cryptosporidium. Trends Parasitol 2008, 24:509-516.

5. Tzipori S, Griffiths JK: Natural history and biology of Cryptosporidium parvum. Adv Parasitol 1998, 40:5-36.

6. Borowski H, Thompson RCA, Armstrong T, Clode PL: Morphological characterization of Cryptosporidium parvum life-cycle stages in an in vitro model system. Parasitology 2010, 137:13-26.
7. Hijjawi N, Estcourt A, Yang R, Monis P, Ryan U: Complete development and multiplication of Cryptosporidium hominis in cell-free culture. Vet Parasitol 2010, 169:29-36.

8. Hijjawi NS, Meloni BP, Ng'anzo M, Ryan UM, Olson ME, Cox PT, Monis PT, Thompson RCA: Complete development of Cryptosporidium parvum in host cell-free culture. Int J Parasitol 2004, 34:769-777.

9. Hijjawi NS, Meloni BP, Ryan UM, Olson ME, Thompson RCA: Successful in vitro cultivation of Cryptosporidium andersoni: evidence for the existence of novel extracellular stages in the life cycle and implications for the classification of Cryptosporidium. Int J Parasitol 2002, 32:1719-1726.

10. Karanis P, Kimura A, Nagasawa H, Igarashi I, Suzuki N: Observations on Cryptosporidium life cycle stages during excystation. J Parasitol 2008, 94:298-300.

11. Rosales MJ, Cordón GP, Moreno MS, Sánchez CM, Mascaró C: Extracellular like-gregarine stages of Cryptosporidium parvum. Acta Trop 2005, 95:74-78.

12. Zhang $L$, Sheoran AS, Widmer G: Cryptosporidium parvum DNA replication in cell-free culture. J Parasitol 2009, 95:1239-1242.

13. Carreno RA, Martin DS, Barta JR: Cryptosporidium is more closely related to the gregarines than to coccidia as shown by phylogenetic analysis of apicomplexan parasites inferred using small-subunit ribosomal RNA gene sequences. Parasitol Res 1999, 85:899-904.

14. Leander BS, Harper JT, Keeling PJ: Molecular phylogeny and surface morphology of marine aseptate gregarines (Apicomplexa): Selenidium spp. and Lecudina spp. J Parasitol 2003, 89:1191-1205.

15. Abrahamsen MS, Templeton TJ, Enomoto S, Abrahante JE, Zhu G, Lancto CA, Deng M, Liu C, Widmer G, Tzipori S, et al: Complete genome sequence of the apicomplexan, Cryptosporidium parvum. Science 2004, 304:441-445.

16. Declerck P, Behets J, Margineanu A, van Hoef V, De Keersmaecker B, Ollevier F: Replication of Legionella pneumophila in biofilms of water distribution pipes. Microbiol Res 2009, 164:593-603.

17. Dunne WM: Bacterial adhesion: seen any good biofilms lately? Clin Microbiol Rev 2002, 15:155-166.

18. Fisher I, Angles M, Chandy J, Cox P, Warnecke M, Kastl G, Jegatheesan V: Biofilms - a sticky situation for drinking water? Water 2000, 27:33-37.

19. Wingender J, Flemming HC: Biofilms in drinking water and their role as reservoir for pathogens. Int J Hyg Environ Health 2011, 214:417-423.

20. Helmi K, Skraber S, Gantzer C, Willame R, Hoffmann L, Cauchie H-M: Interactions of Cryptosporidium parvum, Giardia lamblia, vaccinal poliovirus type 1 , and bacteriophages phiX174 and MS2 with a drinking water biofilm and a wastewater biofilm. Appl Environ Microbiol 2008, 74:2079-2088.

21. Searcy KE, Packman Al, Atwill ER, Harter T: Capture and retention of Cryptosporidium parvum oocysts by Pseudomonas aeruginosa biofilms. Appl Environ Microbiol 2006, 72:6242-6247.

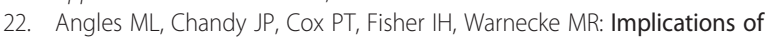
biofilm-associated waterborne Cryptosporidium oocysts for the water industry. Trends Parasitol 2007, 23:352-356.

23. Howe AD, Forster S, Morton S, Marshall R, Osborn KS, Wright P, Hunter PR: Cryptosporidium oocysts in a water supply associated with a cryptosporidiosis outbreak. Emerg Infect Dis 2002, 8:619-624.

24. Meloni BP, Thompson RC: Simplified methods for obtaining purified oocysts from mice and for growing Cryptosporidium parvum in vitro. J Parasitol 1996, 82:757-762.

25. Werner E, Roe F, Bugnicourt A, Franklin MJ, Heydorn A, Molin S, Pitts B, Stewart PS: Stratified growth in Pseudomonas aeruginosa biofilms. Appl Environ Microbiol 2004, 70:6188-6196.

26. Heydorn A, Nielsen AT, Hentzer M, Sternberg C, Givskov M, Ersboll BK, Molin S: Quantification of biofilm structures by the novel computer program COMSTAT. Microbiology 2000, 146(Pt 10):2395-2407.

27. Barraud N, Storey MV, Moore ZP, Webb JS, Rice SA, Kjelleberg S: Nitric oxide-mediated dispersal in single- and multi-species biofilms of clinically and industrially relevant microorganisms. Microb Biotechnol 2009, 2:370-378.

28. Boxell A, Hijjawi N, Monis $\mathrm{P}$, Ryan U: Comparison of various staining methods for the detection of Cryptosporidium in cell-free culture. Exp Parasitol 2008, 120:67-72.

29. Edwards $\mathrm{H}$, Andrew Thompson $\mathrm{R}$, Koh WH, Clode PL: Labeling surface epitopes to identify Cryptosporidium life stages using a scanning electron microscopy-based immunogold approach. Mol Cell Probes 2012, 26:21-28. 
30. Davies DG, Parsek MR, Pearson JP, Iglewski BH, Costerton JW, Greenberg EP: The involvement of cell-to-cell signals in the development of a bacterial biofilm. Science 1998, 280:295-298.

31. Sauer K, Camper AK, Ehrlich GD, Costerton JW, Davies DG: Pseudomonas aeruginosa displays multiple phenotypes during development as a biofilm. J Bacterio/ 2002, 184:1140-1154.

32. Wolyniak EA, Hargreaves BR, Jellison KL: Retention and release of Cryptosporidium parvum oocysts by experimental biofilms composed of a natural stream microbial community. Appl Environ Microbiol 2009, 75:4624-4626.

33. Wolyniak EA, Hargreaves BR, Jellison KL: Seasonal retention and release of Cryptosporidium parvum oocysts by environmental biofilms in the laboratory. Appl Environ Microbiol 2010, 76:1021-1027.

34. Umemiya R, Fukuda M, Fujisaki K, Matsui T: Electron microscopic observation of the invasion process of Cryptosporidium parvum in severe combined immunodeficiency mice. J Parasitol 2005, 91:1034-1039.

35. Lumb R, Smith $K, O^{\prime}$ Donoghue PJ, Lanser JA: Ultrastructure of the attachment of Cryptosporidium sporozoites to tissue culture cells. Parasitol Res 1988, 74:531-536.

36. Current $\mathrm{WL}$, Reese NC: A comparison of endogenous development of three isolates of Cryptosporidium in suckling mice. J Protozool 1986, 33:98-108.

37. Petry F: Structural analysis of Cryptosporidium parvum. Microsc Microanal 2004, 10:586-601.

38. Matsubayashi M, Ando H, Kimata I, Nakagawa H, Furuya M, Tani H, Sasai K: Morphological changes and viability of Cryptosporidium parvum sporozoites after excystation in cell-free culture media. Parasitology 2010, 137:1861-1866.

39. Karanis $P$, Aldeyarbi HM: Evolution of Cryptosporidium in vitro culture. Int $J$ Parasitol 2011, 41:1231-1242.

40. Alarcón ME, Huang CG, Tsai YS, Chen WJ, Dubey AK, Wu WJ: Life cycle and morphology of Steinina ctenocephali (Ross 1909) comb. nov. (Eugregarinorida: Actinocephalidae), a gregarine of Ctenocephalides felis (Siphonaptera: Pulicidae) in Taiwan. Zool Stud 2011, 50:763-772.

41. Leander BS: Marine gregarines: evolutionary prelude to the apicomplexan radiation? Trends Parasitol 2008, 24:60-67.

42. Jirku M, Valigurova A, Koudela B, Krizek J, Modry D, Slapeta J: New species of Cryptosporidium tyzzer, 1907 (Apicomplexa) from amphibian host: morphology, biology and phylogeny. Folia Parasitol (Praha) 2008, 55:81-94.

43. Valigurová A, Jirků M, Koudela B, Gelnar M, Modrý D, Šlapeta J: Cryptosporidia: Epicellular parasites embraced by the host cell membrane. Int J Parasitol 2008, 38:913-922.

44. King BJ, Hoefel D, Lim SP, Robinson BS, Monis PT: Flow cytometric assessment of distinct physiological stages within Cryptosporidium parvum sporozoites post-excystation. Parasitology 2009, 136:953-966

45. Valdez LM, Dang H, Okhuysen PC, Chappell CL: Flow cytometric detection of Cryptosporidium oocysts in human stool samples. J Clin Microbiol 1997, 35:2013-2017.

46. Vesey G, Griffiths KR, Gauci MR, Deere D, Williams KL, Veal DA: Simple and rapid measurement of Cryptosporidium excystation using flow cytometry. Int J Parasitol 1997, 27:1353-1359.

47. Singleton S, Treloar R, Warren P, Watson GK, Hodgson R, Allison C: Methods for microscopic characterization of oral biofilms: Analysis of colonization, microstructure, and molecular transport phenomena. Adv Dent Res 1997 11:133-149.

48. Rickard AH, Gilbert P, High NJ, Kolenbrander PE, Handley PS: Bacterial coaggregation: an integral process in the development of multi-species biofilms. Trends Microbiol 2003, 11:94-100.

49. Okabe $\mathrm{S}$, Kuroda $\mathrm{H}$, Watanabe $Y$ : Significance of biofilm structure on transport of inert participates into biofilms. Water Sci Technol 1998, 38:163-170

50. Okabe $\mathrm{S}$, Satoh $\mathrm{H}$, Kindaichi T: A polyphasic approach to study ecophysiology of complex multispecies nitrifying biofilms. Methods Enzymol 2011, 496:163-184.

\section{doi:10.1186/1756-3305-6-270}

Cite this article as: Koh et al:: Multiplication of the waterborne

pathogen Cryptosporidium parvum in an aquatic biofilm system. Parasites \& Vectors 2013 6:270

\section{Submit your next manuscript to BioMed Central and take full advantage of:}

- Convenient online submission

- Thorough peer review

- No space constraints or color figure charges

- Immediate publication on acceptance

- Inclusion in PubMed, CAS, Scopus and Google Scholar

- Research which is freely available for redistribution

Submit your manuscript at www.biomedcentral.com/submit
C BioMed Central 